\title{
The use of WaveLight Contoura to create a uniform cornea: 6-month results with subjective patient surveys
}

This article was published in the following Dove Press journal:

Clinical Ophthalmology

\author{
Manoj Motwani' \\ Ronald $\mathrm{Pei}^{2}$ \\ 'LASIK Institute, San Diego, CA, USA; \\ ${ }^{2}$ Precision Outcome Consultants, \\ Fresno, CA, USA
}

Purpose: The aim of this study was to report on the 6-month visual results of Contoura with Layer Yolked Reduction of Astigmatism (LYRA) protocol, as well as the subjective patientreported outcomes (PRO) by standardized survey.

Patients and methods: A retrospective analysis was conducted in 50 consecutive eyes with 6-month results that had bilateral Contoura laser-assisted in situ keratomileusis (LASIK) correction of measured astigmatism and axis using the LYRA protocol. PRO were measured via the Refractive Status and Vision Profile (RSVP) standardized survey. Objective visual results, subjective patient results, and rates of regression were reported.

Results: Most (78\%) of the eyes achieved an uncorrected visual acuity (UCVA) of 20/15 and $98 \%$ of the eyes achieved an UCVA of 20/20 vision after primary correction. After enhancements, the percentage of uncorrected eyes achieving 20/15 vision was 90 and 20/20 vision was 100 . RSVP patient-related outcomes showed that all patients $(100 \%)$ were either satisfied or very satisfied with their vision. Most patients $(21 / 25,84 \%)$ were very satisfied. On a numerical ordinal scale of $1-10$ (where 10 is the best vision), as reported by patients post-operative (post-op), 15 $(60 \%)$ patients reported a vision score of 10 , nine $(36 \%)$ patients reported a score of 9 , and one (4\%) patient reported a score of 8 . Initial correction was accurate on all patients, but nine eyes had refractive error changes due to epithelial masking of higher order aberrations (HOAs) over the follow-up period, a number that was also likely artificially high due to study bias.

Conclusion: Contoura measured axis and astigmatism-eliminated HOA, resulting in a more uniform cornea with an accurate removal of astigmatism, excellent 20/15 and 20/20 visual outcomes, and favorable patient-reported subjective outcomes.

Keywords: astigmatism, WaveLight Contoura, topography-guided ablation, LASIK, cornea, Contoura with LYRA protocol

\section{Introduction}

The use of topographic measured refraction with primary refractive correction (WaveLight Contoura) has been reported on by the author in past manuscripts resulting in the creation of the Layer Yolked Reduction of Astigmatism (LYRA) protocol. ${ }^{1-4}$ The use of this protocol results in a more uniform cornea by removing the anterior elevation of the cornea, linking the correction of the higher order aberration (HOA) layer and the lower order astigmatism layer. This more uniform cornea results in a decreased scatter of light, with patients reporting not only increased clarity of day vision but also increased clarity of night vision and decreased night glare/halos. The improvements in night vision are marked with patients reporting not only minimal starbursts around headlights at night,
Correspondence: Manoj Motwani LASIK Institute, 4520 Executive Drive, Suite 230, San Diego, CA 92121, USA

$\mathrm{Tel}+\mathrm{I} 8585540008$

Email drmmlj@gmail.com 
to virtually no halo around light sources, but also marked decrease in light sensitivity in patients who had it. ${ }^{4}$

Some of this increased patient satisfaction was noted in the United States Food and Drug Administration (FDA) Contoura study conducted by Stulting and Fant, ${ }^{5}$ but that study only used manifest refraction during Contoura correction, a protocol that we feel does not recognize the link between HOA reduction and astigmatism. In our opinion, it was important to analyze these subjective patient results utilizing a standardized patient visual survey and also provide further information on visual outcomes from patients who had Contoura with the LYRA protocol performed. The initial study that we published in May 2017 had 3-month results, and we felt that a longer term study was needed. ${ }^{4}$ This study contains 6-month results post-Contoura with LYRA protocol, as well as postprocedure-standardized visual surveys. These surveys are important to understand the patient's experience and satisfaction levels with the procedure. Although visual results can be scientifically tracked and charted, it is more difficult to understand the patient's experience. There are patients achieving 20/20 or 20/15 vision who are not satisfied, and understanding the patient's experience is critical to the evaluation of a procedure.

\section{Patients and methods}

A retrospective analysis was conducted in 50 primary laser-assisted in situ keratomileusis (LASIK) corrections from 25 patients (surgeries were performed at a single center in San Diego, CA, USA, by the same surgeon [MM]). Astigmatism and axis (according to the LYRA protocol) were measured using the Contoura system with myopia within $-8.00 \mathrm{D}$ and astigmatism not exceeding $-3.00 \mathrm{D}$ (the FDA-approved Contoura range). ${ }^{1}$ The FDA criteria are for primary correction of eyes within the aforementioned range and do not directly specify whether manifest refraction or Contoura-measured refraction is to be used. Twenty-five consecutive patients were selected who satisfied all of the following criteria: 6-month follow-up visual and topographic data, bilateral distance correction (no monovision), correction within the FDAapproved range, and completion of a standardized visual survey. Any patient who did not have all of these data was eliminated, and the next consecutive patient was included in the study. This use of consecutive patients eliminated selection bias. We elected to exclude patients with monovision or concurrent corneal inlay to eliminate the variable of their effect on the subjective visual results. The consecutive patient pool for this study was significantly larger than that we had assumed prior to the study initiation. We initially assumed that 30 to 35 consecutive patients who fit the study criteria would yield 25 patients who had the requisite follow-up and Refractive Status and Vision Profile (RSVP) survey, but the consecutive patient pool that had to be used was 52 patients. This was because many of the patients who were doing well never returned for their 6-month follow-ups and some just did not wish to return to complete the survey if they already had their 6-month visit. This large pool presented a selection bias toward patients who had refractive change after the initial healing period ( $\sim 2$ weeks), as these patients were more likely to return for follow-up due to changes to their vision. As the goal for each eye was plano, these patients would require secondary laser enhancements. In this study we performed secondary corrections on nine eyes (18)\%, a number that would drop to $8.6 \%$ if it was considered across the 52 patients in the overall consecutive cohort. As previously stated, the goal for final correction was plano and we reported any change in refraction (after the initial healing period) $\geq 0.50 \mathrm{D}$ of sphere or astigmatism by 6 months postoperatively.

Excluding monovision patients or patients with corneal inlays excluded most of the older patients in this group, as virtually all of our patients in the presbyopic years want some sort of reading correction, but this allowed us to get subjective visual surveys focused on satisfaction with treatment via Contoura with LYRA protocol without the confounding effects of reading correction.

All eyes were monitored for at least 6 months following surgery using refraction, visual acuity, and Topolyzer ${ }^{\mathrm{TM}}$ VARIO HOAs to evaluate the accuracy and success of the treatment. Patients were given the RSVP, a survey developed by Johns Hopkins University to validate patient-reported outcomes (PRO), and survey results were scored according to the Johns Hopkins documentation. This scoring involved reporting observed proportions and averages conforming directly to the guidelines of the survey. This survey was administered at least 6 months postoperatively. Although the RSVP is also supposed to be administered prior to surgery for comparison purposes to after surgery, our main concern was to understand the patient's experience across a consecutive series of patients after LASIK with Contoura with LYRA protocol, to understand and analyze the post-surgical patient subjective experience. There were difficulties in the use of this survey, as it is not optimized for refractive surgery and is somewhat cumbersome for patients to understand without explanation as to how they should compare their presurgery vision with contacts/glasses and their post-LASIK vision. Although a monitor could be placed at all times with the 
patient while taking the survey, we initially considered that a source of bias in that the patient might give falsely positive answers to please us. Patients had difficulties understanding the changing ordinal scales throughout the questionnaire. Patients were recalled to confirm their scores. We attempted to perform these surveys without biasing the patients in a way to please the authors or survey monitors, and this did require an exceptional amount of effort.

HOA treatment was determined by evaluating the $\mathrm{C} 3$ and $\mathrm{C} 5$ polynomial numbers at the $6.5 \mathrm{~mm}$ zone as determined utilizing the Contoura planning system Zernicke readouts. These were obtained after the elimination of poor quality Topolyzer scans, using only consistent, good quality Topolyzer scans.

All surgeries were performed using a WaveLight EX500 with Contoura and the Topolyzer ${ }^{\mathrm{TM}}$ VARIO (Alcon Inc., Fort Worth, TX, USA). All LASIK flaps were made with the Moria M2 Microkeratome (Moria Ophthalmic Instruments, Doylestown, PA, USA) with $110 \mu$ m calibrated blades from Microspecialties (Microspecialties, Inc., Middletown, CT, USA), or the WaveLight FS200 (Alcon Inc.) femtosecond laser set at $110 \mu \mathrm{m}$ flaps. All surgical planning was performed with the Wavenet Server and the Contoura planning system (LYRA protocol) (Alcon Inc.), which has been described in detail in previous publications. ${ }^{2-4}$ This is due to the fact that the aberration removal layer is correlated with the refraction correction layer. The results of that correlation were calculated using the Contoura system.

The LYRA protocol requires the surgeon to use the Contoura-measured astigmatism and axis and adjust the sphere by the spherical equivalent difference in between the manifest and Contoura-measured astigmatism.

Preoperative evaluations included anterior examination, autorefraction with the Nidek OPD (Nidek Inc., Fremont, CA, USA), tonometry, corrected distance visual acuity (CDVA), manifest/cycloplegic manifest refraction, posterior dilated examination, pachymetry via Pentacam (Oculus Optikgeräte $\mathrm{GmbH}$, Wetzlar, Germany), and topographic analysis with the TopolyzerTM VARIO. Our staff is specifically trained to check visions to see if they exceed 20/20 preoperatively, ie, if the patient's best-corrected vision was $20 / 20$ or $20 / 15$ or $20 / 10$.

High-quality reproducible scans (8-12 scans/eye) were obtained with the TopolyzerTM VARIO. At least four highquality scans with appropriate iris registration and complete data were needed to proceed in surgical planning. Great care was taken to avoid inducing astigmatism when holding the eyelids open during the scans. Blinks were permitted to prevent the ocular surface from drying out.
Patients were excluded if they could not achieve 20/20 vision before surgery, had prior refractive surgery, or were not within the approved treatment parameters for the instrument. Anterior segment abnormalities such as corneal ectasia or keratoconus, recurring eye disease such as severe dry eye, uncontrolled diabetes or hypertension, and pregnancy also disqualified patients from participating in the study.

All patients signed written informed consent forms allowing their data to be used in this study. This study falls under the exemption of the Health and Human Services (HHS) Policy for the Protection of Human Research Subjects 45 CFR 46.101 (b) for retrospective studies, and thus, no Institutional Review Board approval was required. This study also conforms to the Declaration of Helsinki guidelines. There were no safety-related incidents that occurred or were reported to Alcon Inc. or WaveLight during this trial.

\section{Results}

Fifty eyes from 25 patients with at least 6 months of postoperative results for each eye were evaluated for refractive result and visual result and analyzed by Zernicke polynomials to measure aberration reduction. All eyes had the target correction postoperatively of plano.

The average age of patients was 27.04 years, with a range of 19-36 years. There were 10 male patients and 15 female patients. The average spherical equivalent of correction performed was $3.97 \mathrm{D}$, with a range of plano to $-7.50 \mathrm{D}$. The average amount of manifest sphere refracted was $-3.41 \mathrm{D}$, and the average amount of treated sphere was $3.32 \mathrm{D}$. The average amount of astigmatism on manifest-measured refraction was $-1.19 \mathrm{D}$, but this was only measured in 39 eyes. The average amount of treated astigmatism was $1.16 \mathrm{D}$, but astigmatism was treated in all 50 eyes.

All 50 eyes received astigmatism treatment. The mean of the absolute value of the deviation of astigmatic power from manifest to measured was $0.43 \mathrm{D}$ (range $=0.05$ to $-1.26 \mathrm{D}$ ). The mean amount of deviation of axis from manifest to measured was $11.46^{\circ}$ (range $=0-87$ ). Eleven eyes exhibited no astigmatism on manifest refraction but demonstrated astigmatism when measured using Contoura.

A small proportion of these patients will show astigmatism due to the epithelium renormalization initially, which fades over a period of a few days to $\sim 2$ weeks. Regression is something that we define as occurring after this initial healing period of the first few weeks. Nine eyes had a change in refraction postoperatively from plano that we corrected for during the first 6 months. Prior to this enhancement, 49 of the $50(98 \%)$ eyes had already achieved 20/20 vision, and 


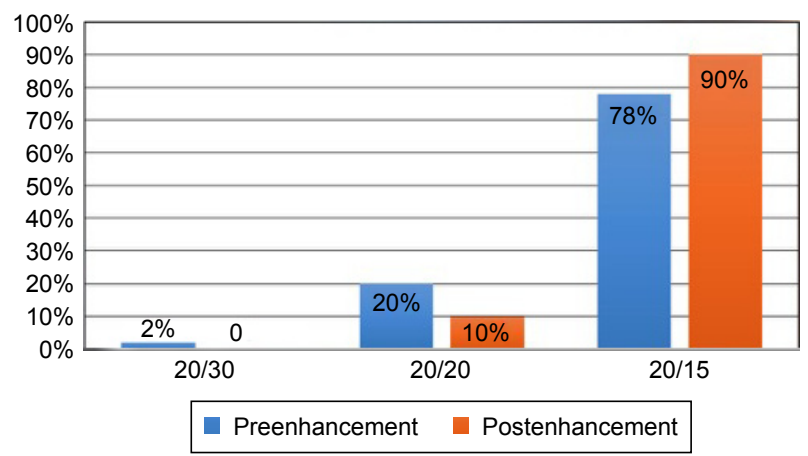

Figure I Uncorrected post-operative vision at 6 months pre-and post-enhancement.

one eye had 20/30 vision (Figure 1). The manifest postoperative refractions of this refractive change are listed in Table 1. The range of spherical equivalent of these eyes was $+0.25 \mathrm{D}$ to $-0.75 \mathrm{D}$, with the absolute value of the mean of the spherical equivalent of $0.51 \mathrm{D}$. All secondary procedures in this study were performed as wavefront optimized corrections (WFO).

Most (39/50; 78\%) of the eyes in this study had achieved $20 / 15$ vision by 6 months without any secondary laser correction. Enhancements were performed as soon as 3 months and as late as 7 months after the primary procedure. Nearly all (49/50; 98\%) eyes achieved 20/20 or better vision, and the remaining eye achieved 20/30 vision prior to enhancement (Figure 1).

Final vision including secondary laser enhancements demonstrated that $45(90 \%)$ of the 50 eyes achieved 20/15 or better vision, with the remaining five eyes achieving 20/20 vision. No eyes in this study finished with $20 / 10$ vision, although a few eyes had 20/10 vision measured at some point during their follow-up. No patient showed any loss of any lines of CDVA vision in this study. Most (45) of the eyes gained a line of vision by the end of the study.

RSVP PRO results are displayed in Figures 2-5. Figure 2 shows the satisfaction with the vision post-op after

Table I Refractions in patients with change from plano correction Post-Contoura with LYRA Protocol

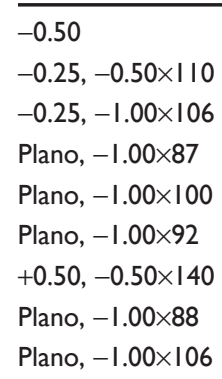

Abbreviation: LYRA, Layer Yolked Reduction of Astigmatism.

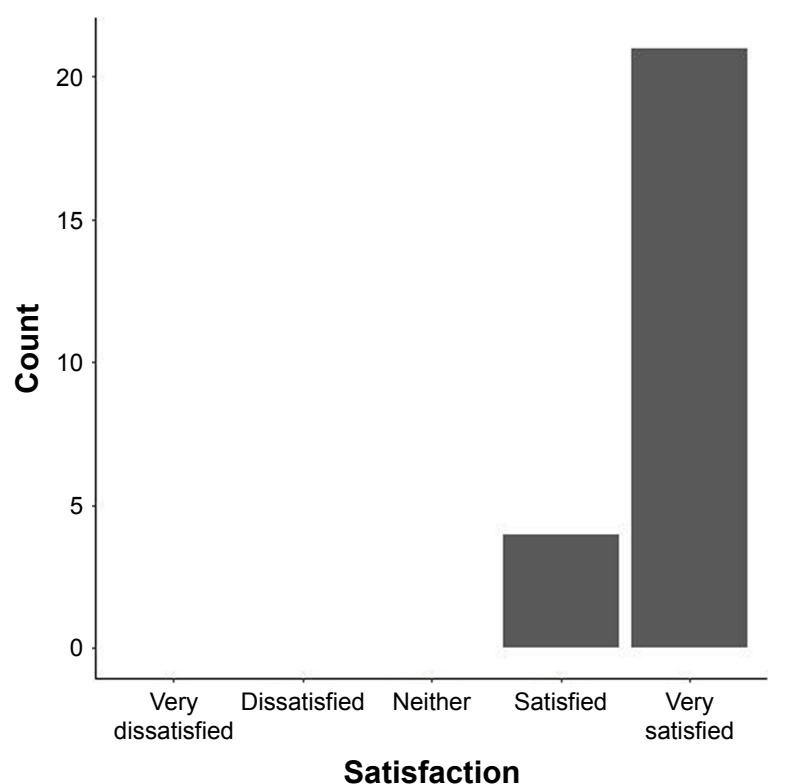

Figure 2 Bar plot of responses to satisfaction with vision in patients who underwent bilateral Contoura LASIK correction of measured astigmatism and axis using the LYRA Protocol.

LASIK via Contoura with LYRA protocol. All patients (100\%) were either satisfied with their vision or very satisfied with their vision. Twenty-one (84\%) patients were very satisfied.

Figure 3 shows a numerical ordinal scale of $1-10$ (where 10 is the best vision) as reported by patients post-op. Fifteen $(60 \%)$ patients reported the vision score of 10 , nine $(36 \%)$ patients reported a score of 9 , and one (4\%) patient reported a score of 8.

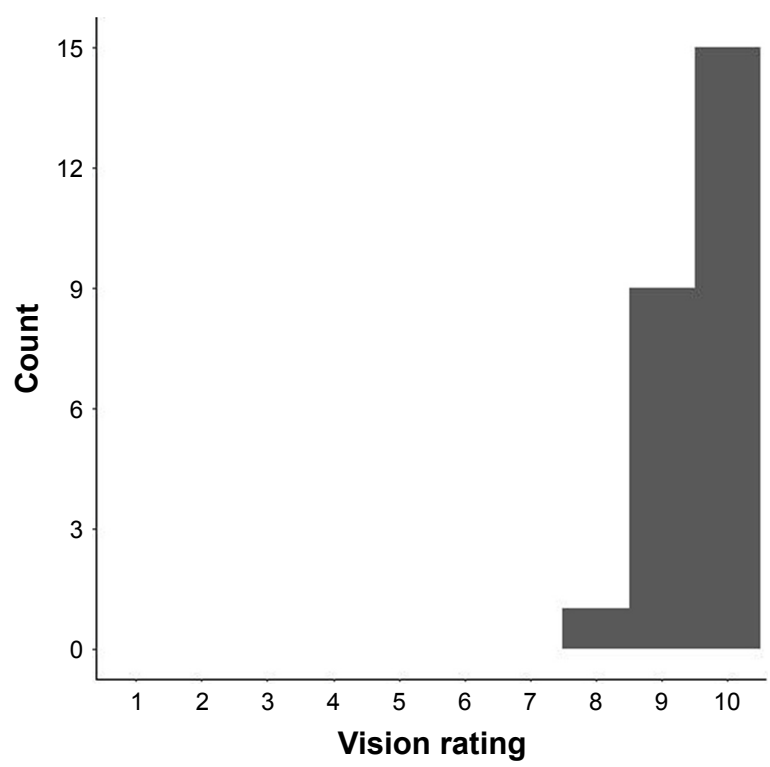

Figure 3 Histogram of numerical rating for vision in patients who underwent bilateral Contoura LASIK correction of measured astigmatism and axis using the LYRA Protocol. 
A

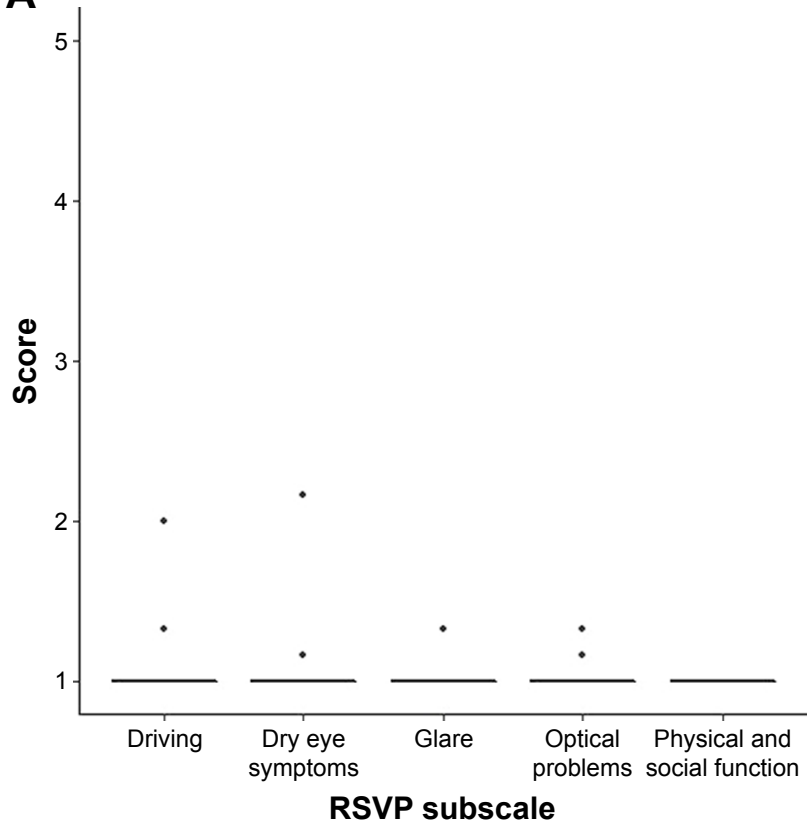

B

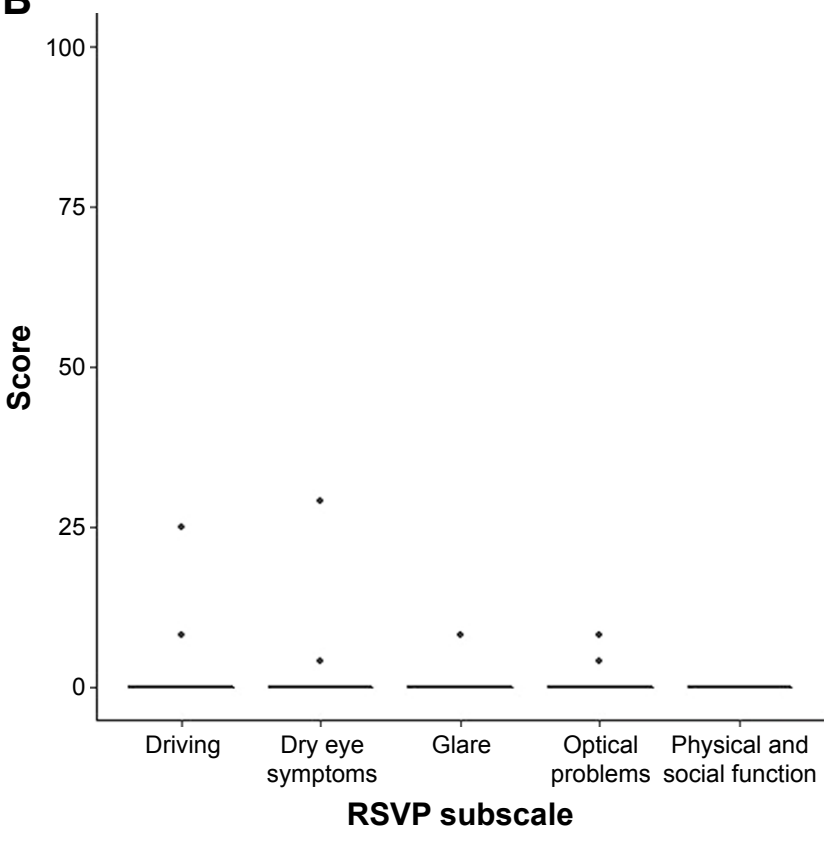

Figure 4 Boxplots for patient RSVP subscale raw scores (A) and scaled scores, according to the RSVP scoring instructions (B). Lower scores represent better satisfaction or fewer problems in that category of daily activities. Higher scores represent higher levels of dissatisfaction or more problems in that area.

Abbreviation: RSVP, Refractive Status and Vision Profile.

Figure 4A and B shows the boxplots quality of vision. These categories specifically zeroed in if patients were having problems with driving, glare, optical problems, physical and social functions, and dry eye symptoms. The lower score translates to better satisfaction or fewer problems in the categories of daily activities. The mean results show that most patients had zero problems, and the points show the outliers in these categories.

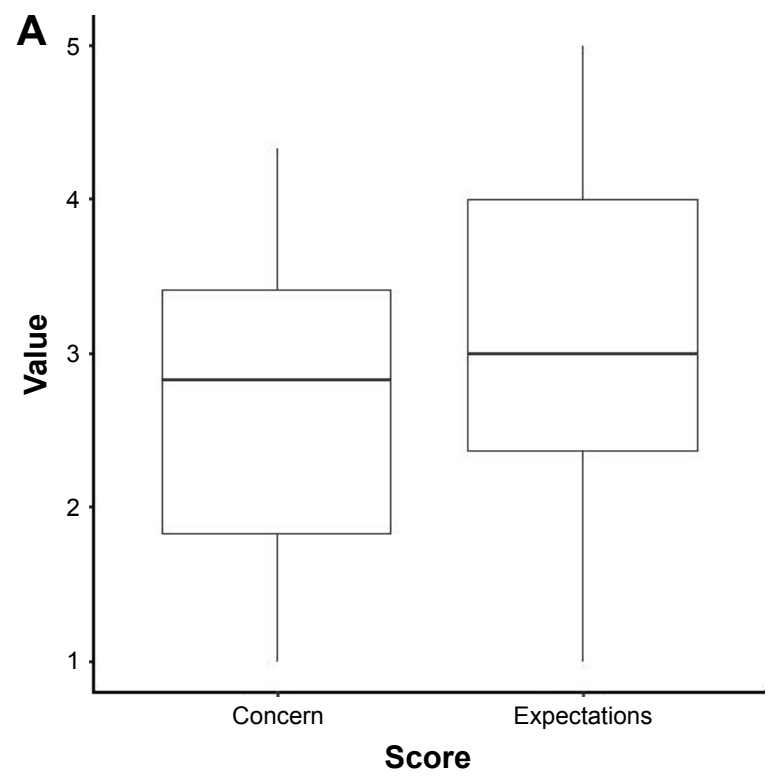

Figure 5A and $\mathrm{B}$ represents the presurgery expectations of LASIK surgery on the patients' eyes and their expectations if they no longer required lenses. The boxplots show that the responses are across the board for both, levels of concern for their vision, and their levels of expectation. In other words, most patients did not have expectations of perfection but wanted their dependence on glasses and contacts reduced and eliminated, if possible.

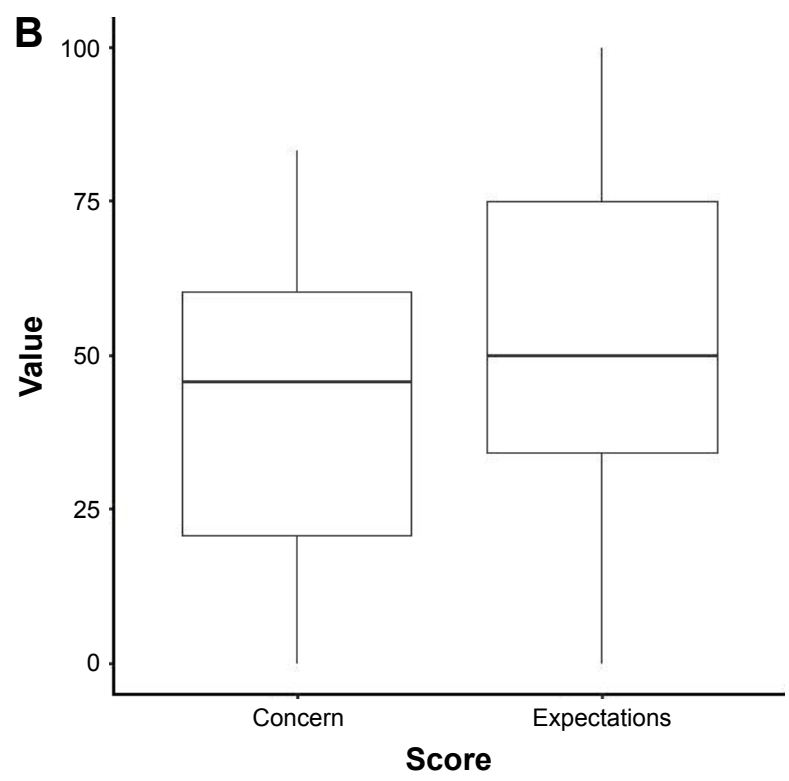

Figure 5 Boxplots on patients' concerns for their vision in general and their expectations if they no longer required lenses on a raw scaling (A) and rescaled according to the RSVP scoring instructions (B). 


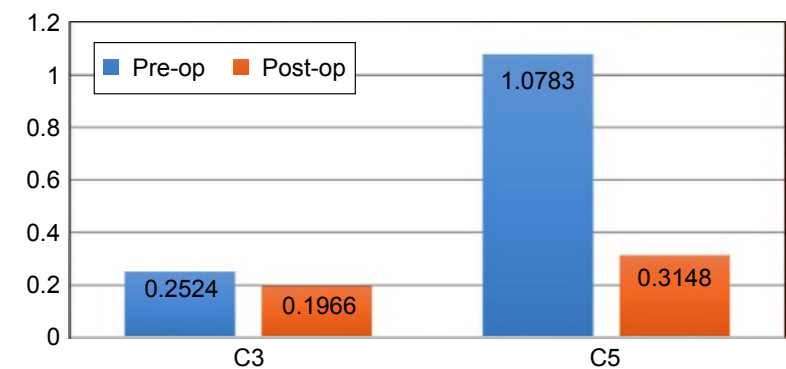

Figure 6 Average $C 3$ and C5 Zernicke polynomial measurements pre- and postoperatively.

Astigmatism polynomials, C3 and C5, were also analyzed pre- and post-op at the $6.5 \mathrm{~mm}$ optical zone. The pre-operative average at $6.5 \mathrm{~mm}$ for C3 was 0.2524 (range 0.8376 to -1.3873 ) and for C5 was 1.0783 (range 0.99 to -3.1153 ). This also demonstrates that the majority of human corneas have with-the-rule astigmatism.

The 6-month post-op average $\mathrm{C} 3$ at $6.5 \mathrm{~mm}$ was 0.1966 (range 0.6075 to -0.6591 ), and the average C5 was 0.3148 (range 0.5621 to -1.3646 ). At 6 months, the average $\mathrm{C} 3$ was reduced by $22 \%$ and the average C 5 was reduced by $70.8 \%$ (Figure 6).

\section{Discussion}

As discussed in the previous studies, ${ }^{2-4}$ the use of the LYRA protocol with WaveLight Contoura is designed to treat the anterior elevation created by the astigmatism and HOAs, thereby creating a more uniform cornea. The manifest refraction is the "point of least visual confusion" between the distortion of the HOA and the distortion of the astigmatism and is post-cortical processing by the patient. The Contoura-measured astigmatism magnitude and axis are what the system determines is present after the corneal HOAs are removed. The use of manifest refraction after the removal of HOA ignores any link between corneal HOA removal and refraction, which can lead to not only inaccurate outcomes but also the formation of new corneal aberrations. Use of the LYRA protocol with Contoura creates a more uniform cornea decreasing scatter of light, with the main limitation being incomplete measurement of the corneal HOA due to epithelial compensation as discussed later in this section.

The visual results from this study were excellent. For primary LASIK procedures, $78 \%$ of eyes achieved $20 / 15$ or better vision, $98 \%$ of eyes achieved $20 / 20$ or better vision, and one ( $2 \%$ ) eye achieved $20 / 30$ vision. If secondary corrections are included, $90 \%$ of patients achieved $20 / 15$ vision and $100 \%$ of patients achieved $20 / 20$ vision (Figure 5 ). The amount of change in refractive error after initial correction in these patients was relatively small, and all save one eye were astigmatic in nature. This is consistent with what we have seen with our clinical experience with Contoura with LYRA protocol in that the regression is almost always astigmatic in nature. ${ }^{3,4}$ These visual results correlate well with our 3 -month results reported in a prior published study, which showed $80.85 \%$ of distance vision eyes achieving $20 / 15$ or better vision at 3 months. ${ }^{4}$

Interestingly, none of the eyes in this group show better than 20/20 vision in the best-corrected preoperative vision testing and all eyes that finished the study at 20/15 vision had gained a line of vision. It is important to note that all patients were checked to determine if they were capable of better than 20/20 best corrected vision during pre-operative undilated refraction.

The subjective visual results show excellent postoperative patient satisfaction. Ninety six percent of patients rated their post-operative visual satisfaction on RSVP survey as a 9 or 10 , and one person scored their satisfaction as an 8 (Figure $2)$. All (100\%) patients reported being satisfied or very satisfied with their vision (Figure 1). More striking was the low amount of postoperative symptoms noted by the patients. When surveyed for comfort driving, glare, optical problems, physical and social functions, and dry eye symptoms postoperatively, the mean patient score in these categories was essentially zero, with one or two outliers per category (Figure 4). These surveys were taken after performing any necessary enhancements, and it is important to note that it has been our clinical experience that after LASIK with Contoura with LYRA protocol, any optical issues that patients complain of can be traced to either regression or dry eye/allergy symptoms. In other words, although we cannot make the corneas fully uniform at this time due to the epithelial masking of HOAs, we do achieve a high enough level of uniformity so that, virtually, all of the optical, comfort, and visual problems we note after these procedures have been treatable. Thus, the results on this postoperative PRO survey are excellent.

Although it has been normal to have preoperative RSVP surveys for comparison purposes in past studies, with this unique treatment, our goal was to show the postoperative satisfaction levels by demonstrating the low level of complaints or symptomology while maintaining excellent objective visual outcomes. ${ }^{6-8}$ With previous laser vision treatments, high objective visual outcomes did not necessarily equate to the subjective quality of vision.

Initial corrections were accurate in all 50 eyes, with eyes achieving plano, or very close to plano, within the initial healing period of about 2 weeks after primary correction. Nine eyes 
(18\%) had refractive error changes after initial primary correction. As mentioned in the "Patients and methods" section, we had a set of criteria for inclusion in this retrospective study and we wanted a consecutive set of patients who would also be able to complete a subjective patient survey. Unfortunately, getting patients to return for 6-month and later post-op visits was harder than we anticipated. We found that older patients were more willing to return, but the younger population ( $<40$ years) seems to be somewhat more transient and less reliable for follow-up examinations unless they have an issue. We expected to be able to use a consecutive series of perhaps 35 patients to yield the desired sample size of 25 patients and 50 eyes. Unexpectedly, we had to increase the consecutive series to 52 patients to yield the sample size of 25 patients desired. Many patients in this consecutive cohort met the inclusion criteria, but they simply did not return for 6 months of follow-up. Therefore, our consecutive series was biased toward patients who returned because of an issue with their vision, ie, refractive error change. Since our goal was plano correction postoperatively, this led to nine eyes with refractive error change, which had secondary laser correction, of which all but one eye (which was $-0.50 \mathrm{D}$ ) had astigmatic regression. This again is consistent with what we have observed in other studies and in clinical practice, that most refractive change is of the astigmatic sort. Considering overall numbers over the past 2 years in our center, we estimate that $\sim 6 \%-7 \%$ of patients will change from plano correction in the 3-6 months after Contoura with LYRA protocol. As we have theorized, and experienced clinically, this is due to epithelial masking of HOA. The refractive change postoperatively from this masking and, therefore, incomplete treatment of HOAs occur after the initial healing period across several months as the epithelium renormalizes to the new corneal stromal shape.

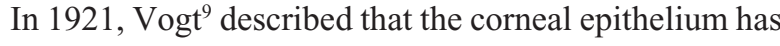
the ability to alter its thickness profile in an attempt to reestablish a smooth, symmetrical optical surface and either partially or totally mask the presence of an irregular stromal surface. Reinstein et al, ${ }^{10,11}$ have utilized the Artemis very high-frequency (VHF) digital ultrasound scan (ArcScan Inc, Morrison, CO, USA) to provide pachymetric maps of the individual corneal layers. This work has demonstrated that epithelium hyperplasia can mask a significant amount of corneal irregularity. ${ }^{10}$ We have already noted, in the LYRA protocol studies of Contoura topography-guided ablation, that the majority of HOA are corneal in nature and the epithelium will mask the irregularity caused by the HOA. Since topography can only measure the anterior corneal surface, this masking of HOA would result in incomplete treatment of the anterior elevations caused by the HOA, and as the epithelium renormalizes, the incomplete treatment results in residual HOA that manifest as astigmatic refractive change. Examination of post-op primary correction topographies of patients with post-op refractive change demonstrates elimination of bowtie astigmatism on the topography. Due to the risk of incomplete measurement yet again of corneal HOAs because of epithelial masking, we clinically have decided to treat using WFO for secondary laser corrections. This achieves plano correction but can induce new regular bowtie astigmatism from the treatment. Due to the small number of these corrections, patients appear to have few problems processing the resulting optical aberrations and are happy with their vision, as our outcome analyses demonstrate.

We are currently studying the phenomenon of epithelial masking of HOA post-Contoura with LYRA protocol with the optical coherence tomography (OCT)-based epithelial mapping system developed by Optovue in conjunction with the company. They recently received the first FDA approval for an OCT-based epithelial thickness mapping system. This work will be reported in further studies.

This epithelial compensation of HOAs is also reflected in the incomplete elimination of $\mathrm{C} 3$ and $\mathrm{C} 5$ polynomials, which denote astigmatism. At 6 months, the reduction of $\mathrm{C} 3$ was only $22 \%$, but preoperative $\mathrm{C} 3$ was quite low. The reduction in C5 was more significant at $70.8 \%$, but it is notable that both average $\mathrm{C} 3$ and $\mathrm{C} 5$ ended up at a relatively low number postoperatively (Figure 6). Epithelial masking of HOAs and anterior elevations due to epithelial compensation/ hyperplasia will affect the final amount of astigmatism that can be calculated via topography and leave some residual astigmatism behind. In other words, if the epithelium is masking some of the aberrations as we have theorized, elimination of all astigmatism bringing the $\mathrm{C} 3$ and $\mathrm{C} 5$ values to 0 may not be possible.

Future work on improving results and decreasing the rate of post-op refractive change would likely need to focus on how to integrate epithelial mapping/basement membrane mapping data into Contoura, likely via some form of highresolution OCT mapping. This creates an interesting scenario, as the initial results from Contoura with LYRA protocol have been excellent, but the ability to fully remove the epithelial masked aberrations would not only decrease light scatter and increase visual clarity but also likely decrease retreatments (enhancements). ${ }^{2-4}$ This effect is likely not just limited to Contoura with LYRA protocol but would also be present in any procedure such as ray tracing that uses the Gullstrand 
model as a fixed eye model, as variability imparted by the surface epithelial layer is not acknowledged.

This study is limited in the sample size being only 50 eyes, as a larger sample size could provide more consistent or less-biased results. Ideally, presenting the RSVP surveys preoperatively as well as postoperatively could also yield a more meaningful result, as a baseline of patient's satisfaction would be present to compare postoperative results. That said, our express intention in using the survey was to measure postoperative satisfaction and we believe that it does represent our overall clinical experience with Contoura with LYRA protocol. Finally, the limitations of patient's follow-up also biased the study toward more patients with regression as they had problems they needed to return for. Upon treatment of this issue, the patients then reported excellent results. Even with these limitations, the data here demonstrate the promise of the Contoura with LYRA protocol procedure, as well as the theory that a more uniform cornea leads to not only excellent objective vision but also excellent patient-reported outcomes.

\section{Conclusion}

Contoura with LYRA protocol using the topographymeasured astigmatism and axis results in excellent objective visual results and excellent patient-reported subjective outcomes. However, a sample size of $>50$ eyes would give a higher CI in support of more definitive conclusions.

\section{Acknowledgments}

The authors thank Sissimos Lemonis of WaveLight GmBH for inspiration, Julie Crider of Collaborative Medical Writing for medical writing, and Crystal Nguyen for biostatistics. This study was performed under an unrestricted grant from Alcon Inc.

\section{Author contributions}

All authors contributed toward data analysis, drafting and revising the paper and agree to be accountable for all aspects of the work.

\section{Disclosure}

MM has received an unrestricted IIT grant for this study from Alcon, Inc. RP is currently an employee of Alcon Laboratories, Inc. However, he was not at the time the study was conducted. The authors report no other conflicts of interest in this work.

\section{References}

1. United States Food and Drug Administration. Premarket Approval (PMA) WaveLight Allegretto Wave Excimer Laser System. Available from: http://www.accessdata.fda.gov/scripts/cdrh/cfdocs/cfpma/pma. cfm?id=p030008. Accessed March 9, 2017.

2. Motwani M. The use of WaveLight ${ }^{\mathbb{1}}$ Contoura to create a uniform cornea: the LYRA Protocol. Part 1: the effect of higher-order corneal aberrations on refractive astigmatism. Clin Ophthalmol. 2017;11:897-905.

3. Motwani M. The use of WaveLight ${ }^{\mathbb{}}$ Contoura to create a uniform cornea: the LYRA Protocol. Part 2: the consequences of treating astigmatism on an incorrect axis via excimer laser. Clin Ophthalmol. 2017;11:907-913.

4. Motwani M. The use of WaveLight ${ }^{\mathbb{E}}$ Contoura to create a uniform cornea: the LYRA Protocol. Part 3: the results of 50 treated eyes. Clin Ophthalmol. 2017;11:915-921.

5. Stulting RD, Fant BS, et al. Results of topography-guided laser in situ keratomileusis custom ablation treatment with a refractive excimer laser. J Cataract Refract Surg. 2016;42(1):11-18.

6. Schein OD. The measurement of patient-reported outcomes of refractive surgery: the refractive status and vision profile. Trans Am Ophthalmol Soc. 2000;98:439-469.

7. Garamendi E, Pesudovs K, Stevens MJ, Elliott DB. The Refractive Status and Vision Profile: evaluation of psychometric properties and comparison of Rasch and summated Likert-scaling. Vision Res. 2006; 46(8-9):1375-1383.

8. Kadkhoda A, Ahani IA, Montazeri A. The Refractive Status and Vision Profile (RSVP): Translation into Persian, reliability and validity. Ophthalmic Epidemiol. 2006;13(6):385-392.

9. Vogt A. Textbook and Atlas of Slit Lamp Microscopy of the Living Eye. Bonn, Germany: Wayenborgh Editions; 1981.

10. Reinstein DZ, Archer TJ, Gobbe M. Refractive and topographic errors in topography-guided ablation produced by epithelial compensation predicted by 3D Artemis VHF digital ultrasound stromal and epithelial thickness mapping. J Refract Surg. 2012;28(9):657-663.

11. Reinstein DZ, Archer TJ, Gobbe M, Silverman RH, Coleman DJ. Epithelial thickness in the normal cornea: three-dimensional display with Artemis very high-frequency digital ultrasound. J Refract Surg. 2008; 24(6):571-581.
Clinical Ophthalmology

\section{Publish your work in this journal}

Clinical Ophthalmology is an international, peer-reviewed journal covering all subspecialties within ophthalmology. Key topics include: Optometry; Visual science; Pharmacology and drug therapy in eye diseases; Basic Sciences; Primary and Secondary eye care; Patient Safety and Quality of Care Improvements. This journal is indexed on Submit your manuscript here: http://www.dovepress.com/clinical-ophthalmology-journal

\section{Dovepress}

PubMed Central and CAS, and is the official journal of The Society of Clinical Ophthalmology (SCO). The manuscript management system is completely online and includes a very quick and fair peer-review system, which is all easy to use. Visit http://www.dovepress.com/ testimonials.php to read real quotes from published authors. 